\title{
A versatile, efficient strategy for assembly of multi-fragment expression vectors in Saccharomyces cerevisiae using 60 bp synthetic recombination sequences
}

\author{
Niels GA Kuijpers ${ }^{1,2}$, Daniel Solis-Escalante ${ }^{1,2}$, Lizanne Bosman ${ }^{1,2}$, Marcel van den Broek ${ }^{1,2}$, Jack T Pronk ${ }^{1,2,3}$,
} Jean-Marc Daran ${ }^{1,2,3}$ and Pascale Daran-Lapujade ${ }^{1,2^{*}}$

\begin{abstract}
Background: In vivo recombination of overlapping DNA fragments for assembly of large DNA constructs in the yeast Saccharomyces cerevisiae holds great potential for pathway engineering on a small laboratory scale as well as for automated high-throughput strain construction. However, the current in vivo assembly methods are not consistent with respect to yields of correctly assembled constructs and standardization of parts required for routine laboratory implementation has not been explored. Here, we present and evaluate an optimized and robust method for in vivo assembly of plasmids from overlapping DNA fragments in S. cerevisiae.

Results: To minimize occurrence of misassembled plasmids and increase the versatility of the assembly platform, two main improvements were introduced; i) the essential elements of the vector backbone (yeast episome and selection marker) were disconnected and ii) standardized 60 bp synthetic recombination sequences nonhomologous with the yeast genome were introduced at each flank of the assembly fragments. These modifications led to a 100 fold decrease in false positive transformants originating from the backbone as compared to previous methods. Implementation of the $60 \mathrm{bp}$ synthetic recombination sequences enabled high flexibility in the design of complex expression constructs and allowed for fast and easy construction of all assembly fragments by PCR. The functionality of the method was demonstrated by the assembly of a $21 \mathrm{~kb}$ plasmid out of nine overlapping fragments carrying six glycolytic genes with a correct assembly yield of 95\%. The assembled plasmid was shown to be a high fidelity replica of the in silico design and all glycolytic genes carried by the plasmid were proven to be functional.

Conclusion: The presented method delivers a substantial improvement for assembly of multi-fragment expression vectors in S. cerevisiae. Not only does it improve the efficiency of in vivo assembly, but it also offers a versatile platform for easy and rapid design and assembly of synthetic constructs. The presented method is therefore ideally suited for the construction of complex pathways and for high throughput strain construction programs for metabolic engineering purposes. In addition its robustness and ease of use facilitate the construction of any plasmid carrying two or more genes.
\end{abstract}

Keywords: In vivo assembly, Saccharomyces cerevisiae, Synthetic biology, Pathway engineering, Homologous recombination

\footnotetext{
* Correspondence: p.a.s.daran-lapujade@tudelft.nl

'Department of Biotechnology, Delft University of Technology, Julianalaan 67, Delft 2628 BC, The Netherlands

${ }^{2}$ Kluyver Centre for Genomics of Industrial Fermentation, PO Box 5057, Delft 2600 GA, The Netherlands

Full list of author information is available at the end of the article
} 


\section{Background}

Restriction and ligation, complemented with the creative application of PCR, has long been the universal method for gene cloning in fundamental research and metabolic engineering [1,2]. However, the increasing size and complexity of today's constructs in metabolic engineering has made design and construction of plasmids by these classical techniques increasingly complicated and time consuming. Several in vitro techniques have been developed to deal with these issues. Methods such as SLIC [3], InFusion ${ }^{\mathrm{Tm}}$ [4], and Gibson's isothermal assembly [5], enable efficient assembly of up to six overlapping DNA fragments into a plasmid. However, the efficiencies of these systems decrease at higher numbers of fragments and commercial kits are required to obtain the necessary recombinases. In contrast, in vivo assembly of multiple overlapping DNA fragments by homologous recombination in Saccharomyces cerevisiae does not exhibit these limitations [6-9]. In this method, yeast is transformed with a mixture of multiple linear DNA fragments, which assemble through homologous recombination of overlapping terminal sequences [10]. Although pioneering work in the 1980s already made use of this method to assemble circular plasmids [11], its application remained limited, probably due to the difficulties in the generation of the terminal homologous sequences required for recombination of the linear fragments. Later, in vivo assembly (also known as transformation associated recombination (TAR)) was used for the cloning of large DNA fragments that were resisting traditional methods based on restriction-ligation [12].

With the development of fast and cost effective chemical DNA synthesis, the method readily took off. It was shown that even single-strand 80 bp 'stitching' oligonucleotides overlapping the ends of adjacent fragments could be used to join DNA sequences by in vivo assembly in S. cerevisiae [13]. The full implication of these developments for TAR cloning was realized when Gibson et al. turned to $S$. cerevisiae to assemble four quarter genomes into a fully synthetic $583 \mathrm{~kb}$ Mycoplasma genitalium genome to overcome the size limitations of in vitro assembly resulting from the requirement of $E$. coli transformation [14]. This successful demonstration led to further research on S. cerevisiae as DNA assembly platform. It was subsequently demonstrated that a whole M. genitalium genome could be successfully assembled out of 25 overlapping DNA fragments in a single step [7]. In follow-up studies it was shown that as many as 38 single-stranded 200 bp oligonucleotides with 20 bp sequence overlaps could be incorporated into a linearized plasmid, thereby creating a whole new platform for gene synthesis [15]. This unparalleled efficiency of homologous recombination in S. cerevisiae, harnessed for high-efficiency in vivo assembly of linear DNA fragments, soon caught the interest of metabolic engineers $[8,9]$. Although TAR cloning showed many advantages, published versions of the method still yield false positive transformants at frequencies ranging from 10 to $80 \%$, an aspect that has hitherto received comparatively little attention [16-18]. One of the main sources of incorrect assembly resides in a high incidence of transformants that contain re-circularized plasmid backbones, which contain all genetic elements required for selection and propagation $[19,20]$. To prevent backbone self-closure, selection procedures based on dual markers and counter-selection have been proposed [20]. Recent published protocols do not adequately deal with this incorrect assembly problem and still rely on single linearized plasmid backbones that are co-transformed to S. cerevisiae with a number of overlapping DNA fragments. Efficiencies measured as the percentage of clones containing the desired plasmid range from $20 \%$ to $90 \%$ and are thought to depend on the length of the homologous regions and the number of fragments to be assembled $[8,9,18]$.

The existing methods show the potential to use in-vivo assembly as a standard tool for assembly of large and complex DNA constructs, but two main points should be addressed to make the system more robust and suited for large scale metabolic engineering; (i) the presence of undesired subassemblies due to regeneration of the plasmid should be reduced; and (ii) in vivo assembly systems should be designed in such way that replacing or swapping fragments should be feasible without extensive DNA modifications.

To meet the above requirements, the aim of the present study was to reduce the incidence of incorrect plasmid assembly and to make a robust, versatile in vivo assembly strategy for multi-component plasmids. To this end, the concept of a single linearized vector backbone was abandoned and replaced by separated key genetic elements involved in plasmid selection and propagation. Furthermore, specially designed 60 bp synthetic homologous recombination sequences (SHR-sequences) were implemented to enhance the versatility of the method. As a proof of principle, the method was used to assemble a $21 \mathrm{~kb}$ plasmid from 9 overlapping fragments, using only PCR and yeast transformation. Key factors for successful and highly efficient assembly of DNA by homologous recombination in S. cerevisiae are discussed.

\section{Results}

The use of single-fragment plasmid backbones results in frequent incorrect assembly

Current in vivo plasmid assembly methods in yeast use a linearized vector containing two elements essential for survival; i) an episome (centromere plus autonomously replicating sequence (CEN/ARS) or 2-micron origin) and ii) one or more selection marker genes. Presence of 
these 'survival elements', essential for replication and selection of the plasmid in yeast, on a single fragment, implies that re-circularization of this fragment will always generate a plasmid conferring viability to transformants in selective medium, and therefore in false positives. Circularization of such plasmid backbones can occur via two mechanisms: homologous recombination and nonhomologous end joining (NHEJ) [21,22]. In the case of homologous recombination, the open end of the backbone recombines with a homologous region present in the backbone itself, which can be as short as 15 bp [23].

To estimate the frequency of false-positive transformants resulting from utilization of linearized plasmid backbones, we took plasmid backbones used in two recently reported $S$. cerevisiae based in vivo assembly methods $[17,18]$. Both methods made use of backbones derived from the plasmid pRS416, although in one BamHI was used to linearize pRS416, leading to a 4898 bp backbone and the other used a 2064 bp backbone resulting from the digestion of pRS416 with SspI. Yeast transformations, using the same amount of linearized plasmid DNA as described in the previous studies (100 fmol), led to over 1000 and $245 \pm 14$ transformants with backbone DNA restricted by BamHI and the SspI respectively (Figure 1). The higher number of clones obtained with BamHI could be explained by circularization of the backbone by cohesive-end ligation of the BamHI-cut DNA, while SspI leaves blunt ends [24]. Furthermore the BamHI fragment was 2.5 fold larger than the SspI fragment, leaving much more chance for circularization by internal recombination on short homologous sequences. These results showed that use of backbones obtained by restriction from standard yeast vectors are a serious factor in determining the fidelity of the system.

\section{Separation of the survival elements is important to reduce plasmid self-closure}

To enhance the versatility of the in vivo assembly platform, we designed specific overlapping sequences (Table 1). These unique synthetic $60 \mathrm{bp}$ homologous recombination sequences (SHR-sequences) were obtained by randomly combining bar-code sequences used in the Saccharomyces Genome Deletion Project (Table 1) [25]. The resulting 60 bp SHR-sequences can be easily attached to any DNA fragment of interest by PCR. The SHR sequences add versatility to the system, thereby creating a platform in which DNA cassettes can be easily interchanged and different combinations of genes can be effortlessly assembled.

In the present study, the classical plasmid backbone was replaced by two separate cassettes flanked by SHR

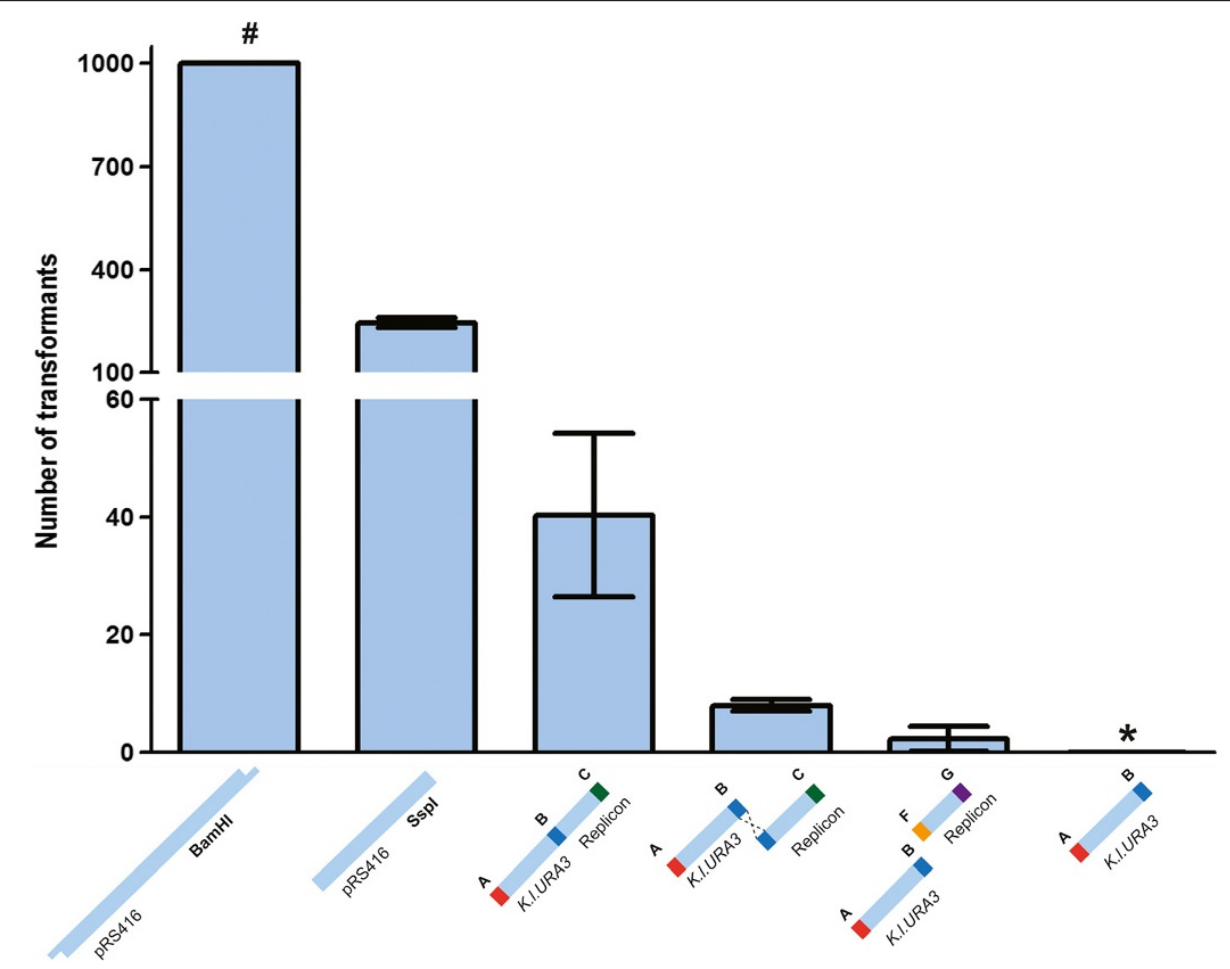

Figure 1 Influence of the plasmid backbone structure on the in vivo assembly efficiency. The quantification of the number of transformants obtained after transformation of $100 \mathrm{fmol}$ of each of the corresponding fragment sets was based on triplicate experiments and the data presented are average \pm standard deviation. (\#) Transformation of the pRS416 backbone linearized by BamHI ( ${ }^{\text {st }}$ bar) gave so many transformants that the exact number of transformants could not be determined due to the colony density on the plates, but it exceeded 1000 transformants in all three transformations. $\left({ }^{*}\right)$ Transformation of the K. lactis URA3 fragment only (rightmost bar) did not yield any transformants. 
Table 1 Overlapping sequences for homologous recombination

\begin{tabular}{|c|c|c|c|c|}
\hline \multirow[b]{2}{*}{ SHR-sequence } & \multirow[b]{2}{*}{ Sequence $5^{\prime} \rightarrow 3^{\prime}$} & \multicolumn{3}{|c|}{$\begin{array}{l}\text { Comparison to S. cerevisiae } \\
\text { CEN.PK113-7D genome }\end{array}$} \\
\hline & & Blast score & E-value & $\%$ GC content \\
\hline \multicolumn{5}{|c|}{ Optimized SHR-sequences } \\
\hline A & ACTATATGTGAAGGCATGGCTATGGCACGGCAGACATTCCGCCAGATCATCAATAGGCAC & 28.3 & 1.3 & 50.0 \\
\hline B & CACCTTTCGAGAGGACGATGCCCGTGTCTAAATGATTCGACCAGCCTAAGAATGTTCAAC & 30.1 & 0.37 & 48.3 \\
\hline C & ACGTCTCACGGATCGTATATGCCGTAGCGACAATCTAAGAACTATGCGAGGACACGCTAG & 26.5 & 4.5 & 50.0 \\
\hline $\mathrm{D}$ & ACGCATCTACGACTGTGGGTCCCGTGGAGAAATGTATGAAACCCTGTATGGAGAGTGATT & 28.3 & 1.3 & 48.3 \\
\hline $\mathrm{F}$ & CATACGTTGAAACTACGGCAAAGGATTGGTCAGATCGCTTCATACAGGGAAAGTTCGGCA & 28.3 & 1.3 & 46.7 \\
\hline G & GCCAGAGGTATAGACATAGCCAGACCTACCTAATTGGTGCATCAGGTGGTCATGGCCCTT & 28.3 & 1.3 & 51.7 \\
\hline $\mathrm{H}$ & AGATTACTCTAACGCCTCAGCCATCATCGGTAATAGCTCGAATTGCTGAGAACCCGTGAC & 30.1 & 0.37 & 48.3 \\
\hline I & TATTCACGTAGACGGATAGGTATAGCCAGACATCAGCAGCATACTTCGGGAACCGTAGGC & 28.3 & 1.3 & 50.0 \\
\hline J & GGCCGTCATATACGCGAAGATGTCCAAGCAGGTAGAACACATAGTCTGAGCATCTCGTCG & 26.5 & 4.5 & 51.7 \\
\hline \multicolumn{5}{|c|}{ Endogenous sequences } \\
\hline A\# & GTCGACAACCCTTAATATAACTTCGTATAATGTATGCTATACGAAGTTATTAGGTCTAGA & 28.3 & 1.3 & 33.3 \\
\hline B\# & GAGTGTTTAGAACATAATCAGTTTATCCATGGTCTATCTCTTCTTGTCGCTITTTCTCCT & 28.3 & 1.3 & 36.7 \\
\hline C\# & TTAATITAAATITITTGGTAGTAAAAGATGCTTATATAAGGATTCGTATTATTGTT & 109 & $5 e-25$ & 18.3 \\
\hline D\# & TAATATTITTCTITTGAAAGTACTACCCACATCCGAACATTGCCACTTACATAGCGATG & 109 & $5 e-25$ & 35.0 \\
\hline J\# & GAACAAAGTATTTAACGCACATGTATAAATATTGTATTAAAAGGGTACCTITATAAATAT & 109 & $5 e-25$ & 23.3 \\
\hline F\# & GAATAGTCTITACACCCACAGTTITCGTGTGGCAGTTACTATATATTAGTAGGATATTC & 109 & $5 e-25$ & 35.0 \\
\hline G\# & CTAAGAAACCATTATTATCATGACATTAACCTATAAAAATAGGCGTATCACGAGGCCCTT & 30.1 & 0.37 & 35.0 \\
\hline $\mathrm{H} \#$ & ACGTAGGATTATTATAACTCAAAAAAATGGCATTATTCTAAGTAAGTTAAATATCCGTAA & 109 & $5 e-25$ & 25.0 \\
\hline I\# & TTGGCAATTाTTGCTCTTCTATATAACAGTTGAAATTTGAATAAGAACATCTTCTCAAA & 109 & $5 e-25$ & 26.7 \\
\hline
\end{tabular}

Sequences were compared to the S. cerevisiae CEN.PK113-7D strain [26] whole genome shotgun contigs (Accession number: PRJNA52955) by BLASTN analysis [27] using BLOSUM 62 substitution matrix. The statistical significance of matches found (Blast score and E-value) are reported.

sequences: one fragment containing the episome and one carrying the selection marker of the plasmid (Figure 2). Since both elements are required for a viable clone and lack any homology to each other, two independent NHEJ events are required to assemble a viable plasmid out of these fragments when co-transformed to $S$. cerevisiae. Moreover, since these fragments are flanked by SHR-sequences, interference and recombination with genomic DNA or internal regions of other assembly cassettes was expected to be less likely.

To quantify the impact of separated survival elements on the occurrence of undesired recombination events, three different cassettes were generated by PCR: (i) A Kluyveromyces lactis URA3 cassette flanked by SHRsequences $A$ and $B$ (K.l.URA $3_{A B}$ ), (ii) a CEN6/ARS4 cassette flanked by SHR-sequences $B$ and $C\left(C E N 6 / A R S 4_{B C}\right)$, and (iii) a CEN6/ARS4 cassette flanked by SHR-sequences $F$ and $G\left(C E N 6 / A R S 4_{F G}\right)$. A fourth cassette was obtained by linking K.l.URA3 $3_{A B}$ and $C E N 6 / A R S 4_{B C}$ by fusion PCR, leading to cassette K.l.URA3/CEN6/ARS4 ${ }_{A C}$. Different fragment combinations containing $100 \mathrm{fmol}$ of each cassette were transformed to the $S$. cerevisiae strain CEN.PK 113-5D, which is auxotrophic for uracil. The fragment K.l. $U R A 3 / C E N 6 / A R S 4_{A C}$, used to mimic a linearized plasmid backbone, was also transformed alone in yeast. As expected, this fragment, which can be circularized in one single NHEJ event, resulted in a substantial number of clones (Figure 1). Still, this short fragment displayed a more than five-fold reduction in clone formation as compared to a linearized backbone (Figure 1). When the overlapping survival elements K.l.URA3 ${ }_{A B}$ and CEN6/ARS4 $4_{B C}$ were co-transformed, a 30 -fold reduction was observed in the number of clones as compared to transformation with linearized backbones (Figure 1). This strong decrease can be explained by the requirement of two recombination events (if integration of the marker in chromosomal DNA is not taken into account) to generate a viable plasmid: the recombination of the separated fragments by homologous recombination and the circularization by NHEJ. When combining the non-overlapping K.l.URA $3_{A B}$ and CEN6/ $A R S 4_{F G}$ fragments hardly any clones $(2 \pm 2)$ were obtained, which is consistent with the need for two recombination events via NHEJ to obtain a viable plasmid. Finally, transformation of only the K.l.URA3 ${ }_{A B}$ cassette did not yield any clones, showing that integration of this cassette in the genome is extremely rare (Figure 1). These results support the hypothesis that separation of the survival elements on non-overlapping fragments reduces plasmid regeneration 


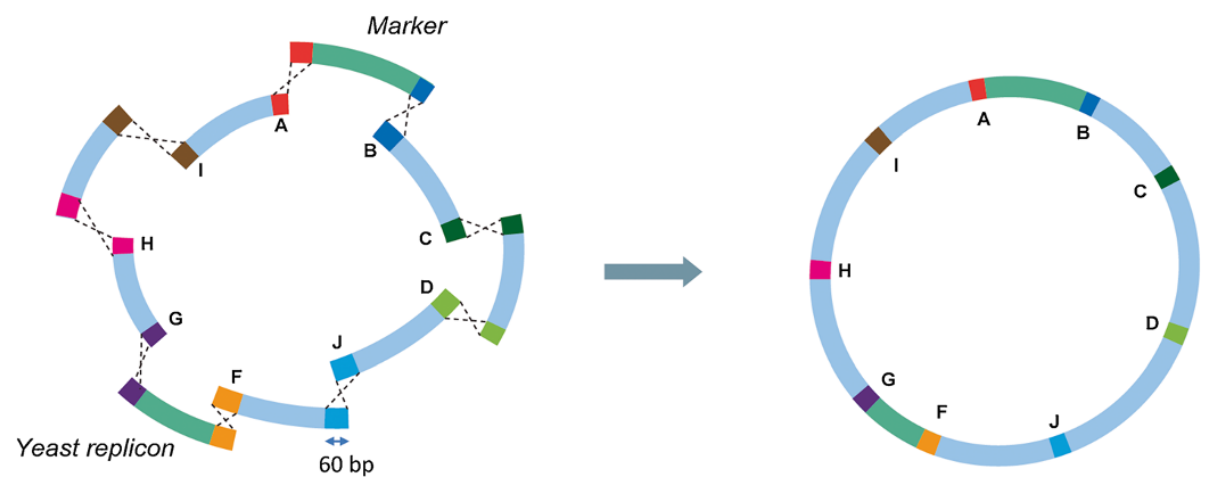

Figure 2 Schematic representation of in vivo assembly of plasmids using 60 bp synthetic homologous recombination sequences. The green survival fragments are essential for replication and selection.

by at least 100 -fold as compared to a linearized plasmid situation (Figure 1). Those results enabled the design of a simple, efficient in vivo assembly platform (Figure 2). All fragments were flanked with $60 \mathrm{bp}$ SHR sequences and two survival elements, both essential for replication and selection of the plasmid, were placed opposite each other in the design.

\section{High efficiency and fidelity of in vivo assembly of a $21 \mathrm{~kb}$ plasmid from nine overlapping fragments}

To test the proposed system, assembly of a $21 \mathrm{~kb}$ plasmid from nine DNA fragments was attempted. The fragments were amplified by PCR to add the desired SHR-sequences designed for recombination of the overlapping fragments. The nine fragments consisted of two $S$. cerevisiae survival elements, an $E$. coli amplification fragment and six expression cassettes, each containing a yeast glycolytic gene fully homologous to its genomic counterpart. The yeast survival elements K.l.URA3 $3_{A B}$ and $C E N 6 / A R S 4_{F G}$ were constructed as described above and the E. coli amplification cassette E.coli IA $_{I A}$ was obtained from pRS416 in the same way. The six glycolytic expression cassettes were amplified by PCR from genomic DNA of $S$. cerevisiae strain CEN.PK113-7D. Based on concentration and size measurements, ca. $100 \mathrm{fmol}$ of the survival elements and $200 \mathrm{fmol}$ of each of the other fragments were pooled and transformed to ca. $10^{8}$ yeast cells. Several controls were performed to estimate the efficiency and reliability of the technique, including: (i) transformation of the marker fragment alone, to estimate the frequency of integration of the fragment into the yeast genome, and (ii) transformation with a control mix in which a single fragment was omitted to estimate the amount of miss-assemblies. After incubation for three days on selective medium, over 1000 clones were obtained for the cells transformed with the complete set of fragments. Conversely, no clones were obtained for the cells transformed with the marker fragment only. The cells transformed with the incomplete control mix yielded six clones. High-fidelity assembly was confirmed by multiplex PCR analysis of 40 clones, randomly picked after two independent transformations. PCR with primers specifically designed to cover the SHR-sequences, produced the expected nine amplicons for 38 out of the 40 clones (Figure 3). Clones obtained by transformation with the incomplete control pool displayed aberrant multiplex PCR profiles. These results provided a strong indication for the presence of the correct assembly in 38 of the 40 tested clones, which corresponded to an extremely high efficiency of correct assembly of $95 \%$.

To ensure that correct multiplex profiles indeed reflected the desired assembly, a single clone was randomly selected and its plasmid was isolated and named pUDC074. After amplification in E. coli, the sequence of pUDC074 was determined by Illumina next generation sequencing and de novo sequence assembly. Sequence analysis confirmed the correct assembly of the plasmid, thereby supporting the practicality of the multiplex PCR approach to screen for correct assemblies. Among the 20,790 bases of pUDC074, as few as four nucleotides were different from the original design. Of these four mutations, three single base-pair deletions were localized in the SHR-sequences. These mutations could result from erroneous homologous recombination in yeast itself, but more likely these errors were introduced prior to assembly during primer synthesis. Although HPLC purified primers were used in this study to attach the SHR-sequences, the use of PAGE purified oligonucleotides could reduce mutations resulting from synthesis. However, since the SHR-sequences are not coding, potential mutations in these overlaps are of minor concern for functionality of the assembly. The single mutation found outside the SHR-sequences was in fragment PFK2 and caused an amino acid substitution in Pfk2 (N822K).

Finally, to determine whether the presence of SHRsequences could have an effect on the biological functionality of the proteins encoded by the six plasmid-borne glycolytic genes, a complementation study was performed 


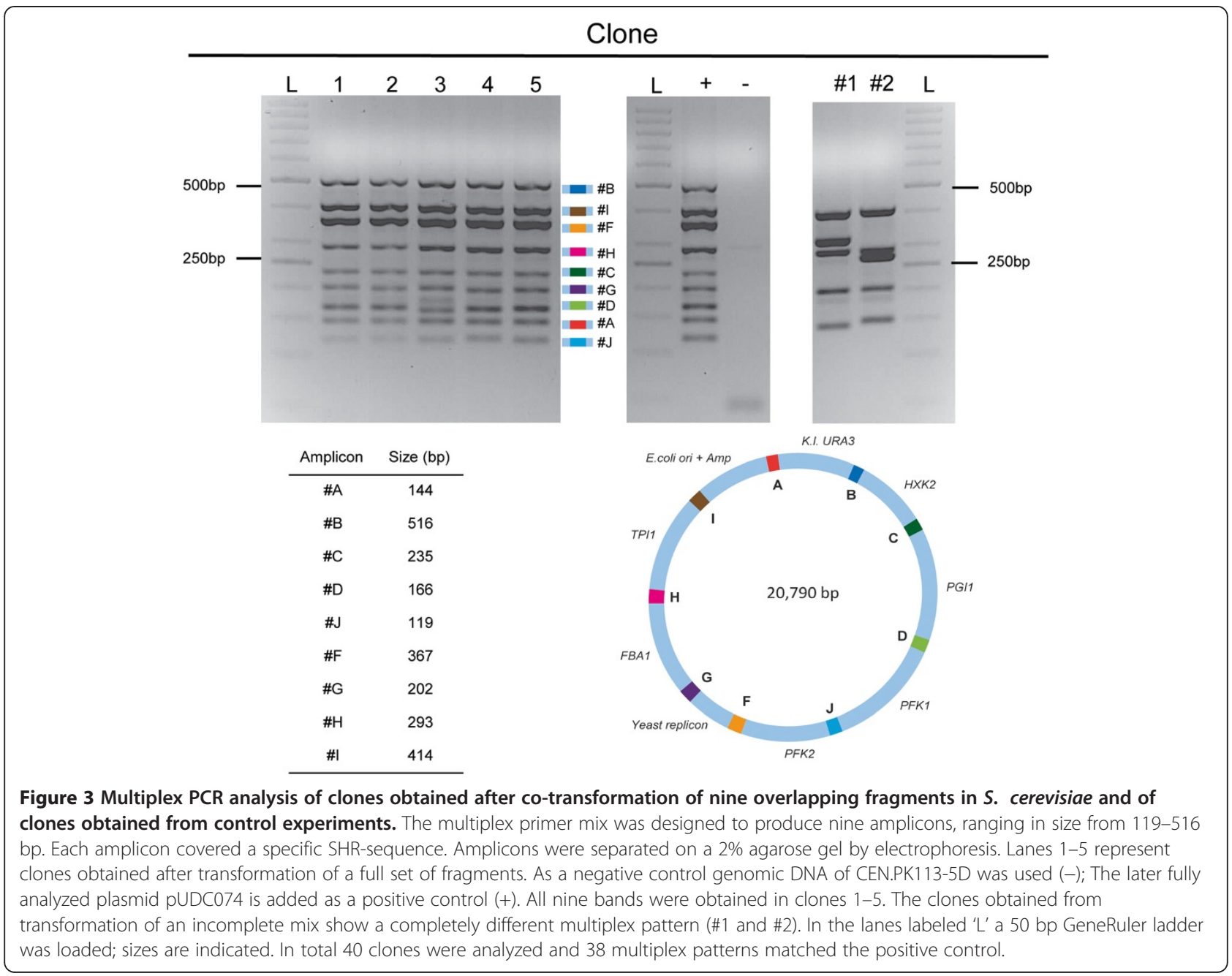

(Figure 4A and B). In S. cerevisiae, deletion of PGI1 [28], TPI1 [29] or FBA1 [30] leads to lethality and deletion of PFK1 or PFK2 results in severe growth impairment [31] when cultivated on glucose. The heterozygous diploids of PGI1, TPI1, FBA1, PFK1 or PFK2 [32] (Table 2) were therefore transformed with the plasmid pUDC074 and subsequently incubated in sporulation medium. After tetrad dissection, the spores containing the deletion could be selected for by the G418 resistance marker $\left(A g T E F 2_{\mathrm{p}^{-}}\right.$ $n p t I I-A g T E F 2_{t}$ ) while the presence of the plasmid was ensured by selecting for the K.l.URA3 marker. The growth of spores in the absence of uracil and in the presence of G418 demonstrated the ability of the plasmid-borne PGI1, TPI1, FBA1, PFK1 and PFK2 genes to complement the deletion of the corresponding chromosomal gene (Figure 4A). Functionality of the assembled HXK2 was demonstrated by restoration of growth on glucose of a glucose phosphorylation-deficient strain (IMX188, $h x k 1$ hxk2 glk1; Table 2) upon transformation with pUDC074. (Figure 4B). These results demonstrated highly efficient assembly of a $21 \mathrm{~kb}$ plasmid out of nine fragments and that the presence of SHR-sequences has no detectable impact on the functionality of the assembled plasmid.

\section{Optimization of SHR-sequences is not critical for high efficiency of in vivo assembly}

In published in vivo assembly studies, the design of the overlapping sequences across which recombination occurs does not receive much attention and often just depends on the ends of the assembled fragments. Therefore, overlaps are likely to differ in GC content and binding capacities could vary strongly between constructs. Moreover, when fragments are specifically designed for metabolic engineering in yeast, overlapping ends often share sequence identity with chromosomal sequences, since expression cassettes typically end with yeast promoter and terminator sequences. The previous experiments demonstrated that combining the separation of essential elements and the use of SHR-sequences resulted in high efficiency of in vivo assembly. To more precisely evaluate the contribution of the customized SHR-sequences on this high efficiency, in vivo assembly was also performed with fragments 


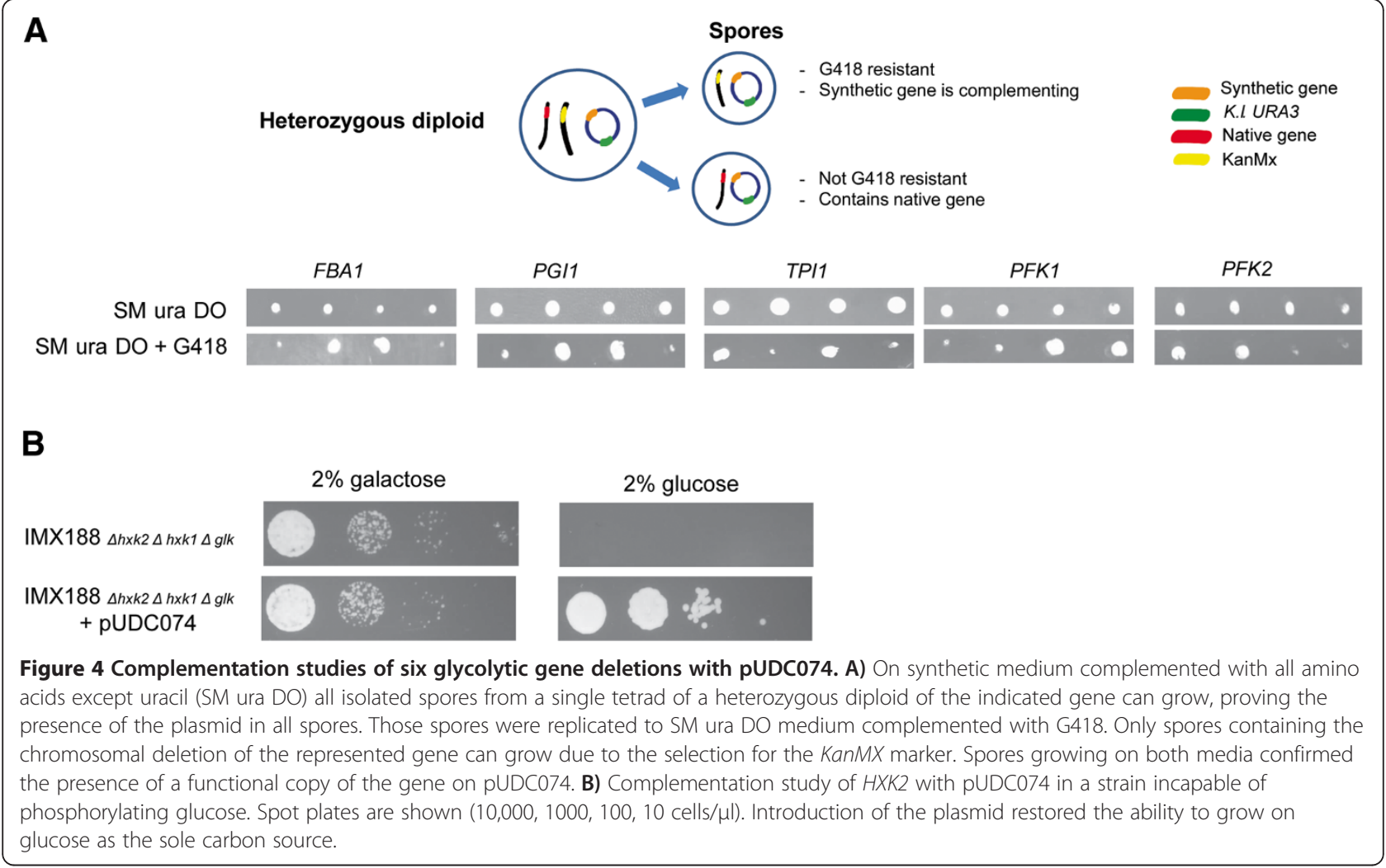

harboring non-optimized overlapping sequences homologous to $S$. cerevisiae genomic DNA with relatively low G/C content $(18.3 \%$ to $36.7 \%$, Table 1$)$. Co-transformation of cassettes carrying the same glycolytic genes as in the previous experiment, but with endogenous overlapping sequences, resulted in similar colony numbers as when SHRsequences were used. In addition, analysis of 10 randomly picked transformants by multiplex PCR did not reveal significant differences in the fidelity of in vivo assembly. These results demonstrated that optimization of the 60 bp overlapping sequences is not required to obtain high in vivo assembly efficiencies with the platform described in this study.

\section{Discussion}

Although uncovered nearly three decades ago [11], the high efficiency of $S$. cerevisiae homologous recombination is only beginning to reveal its full potential for the assembly of large DNA constructs. In vivo assembly in yeast is predicted to have a large impact on laboratory practice, ranging from simple plasmid construction to engineering of complex pathways via automated high-throughput strain construction $[7,8]$. Despite those promising prospects, in vivo assembly has not yet become a standard technique in most academic laboratories. This offers unique possibilities for standardization and, simultaneously, for further optimization. While reported efficiencies of correct assembly of larger (over $15 \mathrm{~kb}$ ) DNA constructs do not exceed $70 \%$, efficiencies of $95 \%$ were reached in the present work for the assembly of a $21 \mathrm{~kb}$ construct $[8,9]$. Physical separation of essential elements of the plasmid backbone contributed to a strong reduction of the frequency of plasmid mis-assembly.

The high efficiency obtained with relatively short 60 bp overlaps demonstrates that, in contrast to practices and claims from recent reports [18,34], longer overlaps are not essential for efficient in vivo assembly. This conclusion is supported by earlier studies in which 30 to 60 bp homologous sequences were shown to lead to high recombination efficiencies in $S$. cerevisiae [32,35]. Although we anticipated that the high GC content, and therefore optimal binding properties, of the optimized SHR-sequences contributed to the high assembly efficiency obtained with the present platform, our results clearly indicate that the nature of these SHR-sequences is not an essential factor for achieving efficiencies above $90 \%$. Therefore the increase in efficiency compared to previous studies essentially originates from the implementation of a backbone-free approach, and more specifically in the physical separation of the genetic elements on a plasmid assembly that are essential for its propagation and selection in the recipient yeast cell. Earlier studies have shown that reassembly of the backbone could make up to $95 \%$ of the obtained clones [20]. 
Table 2 Strains used in this study

\begin{tabular}{|c|c|c|}
\hline Strain & Relevant genotype & Source \\
\hline CEN.PK113-7D & MATa MAL2-8C SUC2 & {$[26,33]$} \\
\hline CEN.PK113-5D & MATa ura3-52 MAL2-8C SUC2 & [33] \\
\hline IMX188 & 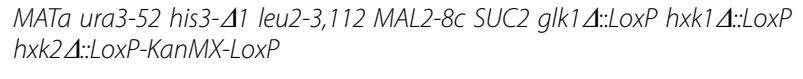 & This study \\
\hline IMX214 & 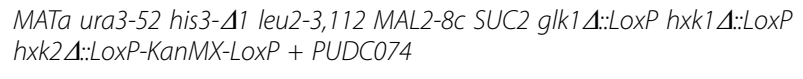 & This study \\
\hline BY4743-Y23336 & 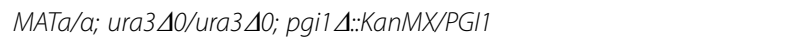 & EUROSCARF $^{\mathrm{a}}$ \\
\hline BY4743-Y25893 & MATa/a; ura3 $\Delta 0 /$ ura3 $\Delta 0 ;$ pfk1 $\Delta:$ KanMXIPFK1 & EUROSCARF \\
\hline BY4743-Y20791 & MATa/a; ura3 $\Delta 0 /$ ura3 $\Delta 0 ;$ pfk2 $2:$ KanMX/PFK2 & EUROSCARF $^{a}$ \\
\hline BY4743-Y23986 & MATa/a; ura3 $\Delta 0 /$ ura3 $\Delta 0$; tpi1 $\Delta:: K a n M X / T P I 1$ & EUROSCARF $^{\mathrm{a}}$ \\
\hline BY4743-Y24909 & MATa/a; ura3 $\triangle 0 /$ ura3 $\Delta 0$; fba $14:$ KanMXIFBA1 & EUROSCARF $^{\mathrm{a}}$ \\
\hline Spore from Y23336 + pUDC074 & $\begin{array}{l}\text { ura340; pgi1::kanMX pUDC074 (KII.URA3(pUG72)TPI HXK2 FBA1 PGI PFK1 } \\
\text { PFK2) }\end{array}$ & This study \\
\hline Spore from Y25893 + pUDC074 & $\begin{array}{l}\text { ura3 10; pfk1:kanMX pUDC074 (KIIURA3(pUG72)TPI HXK2 FBA1 PGI PFK1 } \\
\text { PFK2) }\end{array}$ & This study \\
\hline Spore from Y20791 + pUDC074 & $\begin{array}{l}\text { ura3 10; pfk2::kanMX pUDC074 (KIIURA3(pUG72)TPI HXK2 FBA1 PGI PFK1 } \\
\text { PFK2) }\end{array}$ & This study \\
\hline Spore from Y24909 + pUDC074 & $\begin{array}{l}\text { ura340; tpi1::kanMX pUDC074 (KII.URA3(pUG72)TPI HXK2 FBA1 PGI PFK1 } \\
\text { PFK2) }\end{array}$ & This study \\
\hline Spore from Y23986 + pUDC074 & $\begin{array}{l}\text { ura3 } 40 \text {; fba1::kanMX pUDC074 (KII.URA3(pUG72)TPI HXK2 FBA1 PGI PFK1 } \\
\text { PFK2) }\end{array}$ & This study \\
\hline
\end{tabular}

${ }^{\mathrm{a}}$ http://web.uni-frankfurt.de/fb15/mikro/euroscarf/.

Placing the essential yeast elements on different fragments flanked by SHR-sequences and co-transforming them to $S$. cerevisiae reduced erroneous assemblies by plasmid regeneration by a factor of at least 100 (Figure 1), thereby substantially increasing the fidelity of in vivo assembly. Other yet unidentified factors, such as yeast strain-dependent efficiency of homologous recombination, could also have contributed to the high efficiency of assembly in the present platform and should be considered for further development of the method.

A potential downside of the proposed system was the inherent increase in the number of fragments to be transformed. However, in a standardized transformation protocol, assembly of a plasmid from 16 fragments still generated hundreds of clones (data not shown), which is more than sufficient for metabolic engineering purposes. This result indicates that increasing the number of fragments is not a serious limitation and that use of two survival elements instead of one single backbone does not have a high impact on the overall transformation efficiency.

The considerable potential of $S$. cerevisiae for pathway assembly has been recently proposed $[8,9]$ for short pathways up to eight genes. To obtain highly productive and balanced synthetic pathways it is evident that finding the optimal combination of (heterologous) genes and expressing them at the right levels is essential [36]. Combinatorial approaches are therefore necessary and hundreds to thousands of constructs carrying different alleles expressed behind various promoters will have to be constructed in high throughput platforms. The presented approach will facilitate these strain construction programs, since assembly efficiency and robustness are bound to be key variables for high throughput strain construction, as they determine how many clones will be correctly assembled. The SHR-sequences can be designed and tested for these requirements and thus contribute to the development of these systems. Moreover, the use of SHR-sequences offers an unprecedented versatility. It is a goal of synthetic biology to create versatile platforms with libraries of interchangeable parts and pieces, as exemplified by the BioBricks concept [37]. Using the SHR sequences libraries of standardized parts, ranging from individual gene expression cassettes to fragments that carry entire pathways, can be generated and used for combinatorial assembly and subsequent screening for high-performing strains. In contrast to existing in vivo assembly approaches, no extensive re-designing has to be performed. Libraries of survival elements, genes and SHR-sequences will enable easy in silico design, straightforward in vitro synthesis of the fragments by PCR and efficient in vivo assembly.

While implementing in vivo assembly in our research, we have been surprised by its simplicity, ease of implementation and high efficiency. Within two years, in vivo assembly has almost completely replaced standard restriction/ligation protocols for construction of plasmids 
carrying two or more genes in our laboratory, thereby greatly accelerating strain construction and opening possibilities for strain modification that previously would have been deemed too complicated. Looking into the future, $S$. cerevisiae has the potential to be developed into an even more powerful platform. Similarly to popular $E$. coli strains, which have been extensively optimized to become extremely efficient hosts for plasmid transformation and replication, specific modifications of the yeast chassis, such as removal of the NHEJ machinery or enhancement of DNA uptake, could further extend the efficiency and fidelity of the in vivo assembly method.

\section{Conclusions}

The presented method for in vivo assembly of multifragment expression vectors in Saccharomyces cerevisiae delivers a substantial improvement in terms of fidelity and flexibility as compared to existing methods. This improvement, achieved by replacing the plasmid backbone by standardized survival elements and by implementing the use of standardized 60 bp synthetic recombination sequences, was demonstrated by the correct assembly of a $21 \mathrm{~kb}$ plasmid from nine fragments with an efficiency of $95 \%$. Ideal for the assembly of large constructs, the presented approach delivers a straightforward method for the assembly of any DNA construct carrying two or more genes and can be implemented in any molecular biology laboratory. It is our hope that the present work will contribute to standardization of in vivo assembly of plasmids, artificial chromosomes and synthetic genomes in S. cerevisiae.

\section{Methods}

\section{Strains, media and DNA templates}

The $S$. cerevisiae strains used in this study are listed in Table 2. Cultures for transformation were cultivated in complex media containing $10 \mathrm{~g} \cdot \mathrm{l}^{-1}$ Bacto yeast extract, $20 \mathrm{~g}^{-1} \mathrm{l}^{-1}$ Bacto peptone and $20 \mathrm{~g} \cdot \mathrm{l}^{-1}$ glucose as carbon source. Synthetic medium (SM) contained per liter of demineralized water $2 \%$ agar (w/v) $5 \mathrm{~g}_{(}\left(\mathrm{NH}_{4}\right)_{2} \mathrm{SO}_{4}, 3 \mathrm{~g}$ $\mathrm{KH}_{2} \mathrm{PO}_{4}, 0.5 \mathrm{~g} \mathrm{MgSO}_{4} .7 \cdot \mathrm{H}_{2} \mathrm{O}$, and trace elements according to [38]. Filter-sterilized vitamins were added after heat sterilization of the medium at $120^{\circ} \mathrm{C}$ for 20 $\mathrm{min}$. Glucose or galactose were separately sterilized at $110^{\circ} \mathrm{C}$ and added to a final concentration of $20 \mathrm{~g} \cdot \mathrm{l}^{-1}$. When required, the medium was supplemented with appropriate amounts of auxotrophic requirements [39]. Sporulation medium contained, per liter of demineralized water, $10 \mathrm{~g}$ potassium acetate, $1 \mathrm{~g}$ Bacto yeast extract and $0.5 \mathrm{~g}$ glucose. To rescue strain auxotrophy $10 \mathrm{mg}$ leucine, $5 \mathrm{mg}$ histidine, $5 \mathrm{mg}$ lysine and $5 \mathrm{mg}$ methionine per liter were added prior to heat sterilization. Solid medium was prepared by adding $2 \%$ agar $(\mathrm{w} / \mathrm{v})$ to the media prior to heat sterilization. Spores were grown on solid SM supplemented with Yeast Synthetic Drop-out Medium supplements without uracil (Sigma, St Louis, MO) and replica plated on the same medium supplemented with G418 (200 mg: $\left.\mathrm{l}^{-1}\right)$.

Plasmids pUG72 [40] and pRS416 [41] were maintained in E. coli $\mathrm{DH} 5 \alpha$ and isolated with the GenElute ${ }^{\mathrm{Tn}}$ Plasmid Miniprep Kit (Sigma). Genomic DNA was isolated from $S$. cerevisiae CEN.PK113-7D using the Qiagen 100/G kit (Qiagen, Hilden, Germany).

\section{Production of DNA fragments and transformation}

Fragments for in vivo assembly were obtained from either genomic or plasmid template DNA by extension PCR using Phusion ${ }^{\circledR}$ Hot Start II High Fidelity DNA Polymerase (Thermo Fisher Scientific, Waltham, MA). Primers were HPLC purified (Sigma, St Louis, MO) and are given in Table 3. To improve the PCR efficiency, we modified the conditions recommended by the supplier, by decreasing the primer concentration from $500 \mathrm{nM}$ to $200 \mathrm{nM}$ and increasing the polymerase concentration from $0.02 \mathrm{U} . \mu l^{-1}$ to $0.03 \mathrm{U} . \mu l^{-1}$. All other conditions were chosen according to standard manufacturer instructions. The primers were designed in such a way that the annealing temperature was $>65^{\circ} \mathrm{C}$ to minimize non-specific product formation caused by false priming. Subsequently, the amplified fragments were concentrated by chromatography using Vivacon 500 spin columns (Sartorius Stedim, Aubagne, France). Conversely, fragments obtained from plasmid templates were submitted to an extra purification step by gel extraction to avoid contamination of the fragments by the linearized template plasmid and the ensuing formation of false positive clones. Restriction of pRS416 to obtain the linearized backbones was performed with FastDigest enzymes BamHI and SspI (Thermo Fischer Scientific) according to manufacturer instructions. The fragments CEN6/ARS4 $4_{F G}$ and E.coli $I_{I A}$ were amplified from pRS416. Fragment K.l.URA3 $3_{A B}$ was amplified from pUG72. Fragment K.l.URA3/CEN6/ARS4 ${ }_{A C}$ was obtained by fusion PCR from fragments K.l.URA $3_{A B}$ and $C E N 6 / A R S 4_{B C}$ using primers Fus1 and Fus2. The fragments containing the glycolytic genes were all amplified from CEN.PK113-7D genomic DNA. DNA concentrations were measured by the NanoDrop 2000 spectrophotometer (Thermo Fisher Scientific) and $200 \mathrm{fmol}$ of each glycolytic gene cassette and the E.coli $i_{I A}$ fragment were pooled with $100 \mathrm{fmol}$ of the K.l.URA3 $3_{A B}$ and CEN6/ARS4 $4_{F G}$ fragments in a final volume of $50 \mu \mathrm{l}$. A control pool lacking the PFK2 cassette was created in the same way. Both pools were transformed to S. cerevisiae strain CEN.PK113-5D using the lithium acetate protocol [42]. After transformation cells were selected on synthetic medium for 3-4 days at $30^{\circ} \mathrm{C}$. 
Table 3 Primers used in this study

\begin{tabular}{|c|c|c|}
\hline Primers & Purification & Sequence $5^{\prime} \rightarrow 3^{\prime}$ \\
\hline & & To add SHR-sequences \\
\hline E.coli Rv $+A$ & HPLC & $\begin{array}{l}\text { GTGCCTATTGATGATCTGGCGGAATGTCTGCCGTGCCATAGCCATGCCTTCACATATAGTTGCGCGGAA } \\
\text { CCCCTATTTG }\end{array}$ \\
\hline E.coli Fw +I & HPLC & $\begin{array}{l}\text { TATTCACGTAGACGGATAGGTATAGCCAGACATCAGCAGCATACTTCGGGAACCGTAGGCGAGAGGC } \\
\text { GGTTTGCGTATTGG }\end{array}$ \\
\hline $\mathrm{TPl} 1 \mathrm{Rv}+\mathrm{H}$ & HPLC & $\begin{array}{l}\text { AGATTACTCTAACGCCTCAGCCATCATCGGTAATAGCTCGAATTGCTGAGAACCCGTGACTAGTGTGAG } \\
\text { CGGGATTTAAACTGTG }\end{array}$ \\
\hline TPI1 FW +l & HPLC & $\begin{array}{l}\text { GCCTACGGTTCCCGAAGTATGCTGCTGATGTCTGGCTATACCTATCCGTCTACGTGAATAGCGAAAATGA } \\
\text { CGCTTGCAGTG }\end{array}$ \\
\hline $\mathrm{FBA} 1 \mathrm{R} v+\mathrm{H}$ & HPLC & $\begin{array}{l}\text { GTCACGGGTTCTCAGCAATTCGAGCTATTACCGATGATGGCTGAGGCGTTAGAGTAATCTAAAATCTCAA } \\
\text { AAATGTGTGGGTCATTACG }\end{array}$ \\
\hline$F B A 1 F w+G$ & HPLC & $\begin{array}{l}\text { GCCAGAGGTATAGACATAGCCAGACCTACCTAATTGGTGCATCAGGTGGTCATGGCCCTTAGTGCATGA } \\
\text { CAAAAGATGAGCTAGG }\end{array}$ \\
\hline Cen6 Ars4 Rv $+G$ & HPLC & $\begin{array}{l}\text { AAGGGCCATGACCACCTGATGCACCAATTAGGTAGGTCTGGCTATGTCTATACCTCTGGCGACGGATCG } \\
\text { CTTGCCTGTAAC }\end{array}$ \\
\hline Cen6 Ars4 Fw +F & HPLC & $\begin{array}{l}\text { CATACGTTGAAACTACGGCAAAGGATTGGTCAGATCGCTTCATACAGGGAAAGTTCGGCAGTGCCACCT } \\
\text { GGGTCCTITC }\end{array}$ \\
\hline Cen6 Ars4 Rv +B & HPLC & $\begin{array}{l}\text { CACCTITCGAGAGGACGATGCCCGTGTCTAAATGATTCGACCAGCCTAAGAATGTTCAACGTGCCACCT } \\
\text { GGGTCCTITTC }\end{array}$ \\
\hline Cen6 Ars4 Fw +C & HPLC & $\begin{array}{l}\text { CTAGCGTGTCCTCGCATAGTTCTTAGATTGTCGCTACGGCATATACGATCCGTGAGACGTGACGGATCG } \\
\text { CTTGCCTGTAAC }\end{array}$ \\
\hline PFK2 Rv +F & HPLC & $\begin{array}{l}\text { TGCCGAACTTTCCCTGTATGAAGCGATCTGACCAATCCTTTGCCGTAGTTTCAACGTATGATAGCCATTCT } \\
\text { CTGCTGCTTTGTTG }\end{array}$ \\
\hline PFK2 FW +J & HPLC & $\begin{array}{l}\text { GGCCGTCATATACGCGAAGATGTCCAAGCAGGTAGAACACATAGTCTGAGCATCTCGTCGGAGATCCGA } \\
\text { GGGACGTTTATTGG }\end{array}$ \\
\hline PFK1 Rv +D & HPLC & $\begin{array}{l}\text { ACGCATCTACGACTGTGGGTCCCGTGGAGAAATGTATGAAACCCTGTATGGAGAGTGATTTCGAGATTCCT } \\
\text { CAATCCATACACCATTATAG }\end{array}$ \\
\hline PFK1 FW +J & HPLC & $\begin{array}{l}\text { CGACGAGATGCTCAGACTATGTGTTCTACCTGCTTGGACATCTTCGCGTATATGACGGCCTGTCGTCTTCGT } \\
\text { GAACCATTGTC }\end{array}$ \\
\hline PGl1 Rv +D & HPLC & $\begin{array}{l}\text { AATCACTCTCCATACAGGGTTTCATACATTTCTCCACGGGACCCACAGTCGTAGATGCGTCTGAAGAAGGC } \\
\text { ATACTACGCCAAG }\end{array}$ \\
\hline PGI1 FW $+\mathrm{C}$ & HPLC & $\begin{array}{l}\text { ACGTCTCACGGATCGTATATGCCGTAGCGACAATCTAAGAACTATGCGAGGACACGCTAGTTCGCGACACA } \\
\text { ATAAAGTCTTCACG }\end{array}$ \\
\hline $\mathrm{HXK} 2 \mathrm{Rv}+\mathrm{C}$ & HPLC & $\begin{array}{l}\text { CTAGCGTGTCCTCGCATAGTTCTTAGATTGTCGCTACGGCATATACGATCCGTGAGACGTGCAAGAGAAAAA } \\
\text { AACGAGCAATTGTTAAAAG }\end{array}$ \\
\hline $\mathrm{HXK} 2 \mathrm{FW}+\mathrm{B}$ & HPLC & $\begin{array}{l}\text { CACCTITCGAGAGGACGATGCCCGTGTCTAAATGATTCGACCAGCCTAAGAATGTTCAACGACGGCACCGGG } \\
\text { AAATAAACC }\end{array}$ \\
\hline URA3K.I. RV +B & HPLC & $\begin{array}{l}\text { GTTGAACATTCTTAGGCTGGTCGAATCATTAAGACACGGGCATCGTCCTCTCGAAAGGTGCTCAGAAGCTCAT } \\
\text { CGAACTGTCATC }\end{array}$ \\
\hline URA3K.I. FW + A & HPLC & $\begin{array}{l}\text { ACTATATGTGAAGGCATGGCTATGGCACGGCAGACATTCCGCCAGATCATCAATAGGCACGATCCCAATACA } \\
\text { ACAGATCACGTGATC }\end{array}$ \\
\hline FUS1 & HPLC & ACTATATGTGAAGGCATGGCTATGG \\
\hline FUS2 & HPLC & CTAGCGTGTCCTCGCATAGTTC \\
\hline Amp-rv + A-ctrl & HPLC & $\begin{array}{l}\text { TCTAGACCTAATAACTTCGTATAGCATACATTATACGAAGTTATATTAAGGGTTGTCGACTGCGCGGAA } \\
\text { CCCCTATTTG }\end{array}$ \\
\hline Amp-fw $+|-c t r|$ & HPLC & $\begin{array}{l}\text { TTGGCAATTIITGCTCTTCTATATAACAGTTGAAATTTGAATAAGAACATCTTCTCAAAGAGAGGCGGTTा } \\
\text { GCGTATTGG }\end{array}$ \\
\hline CEN/6ARS4-fw + F-ctrl & HPLC & $\begin{array}{l}\text { GAATAGTCTITACACCCACAGTITITCGTGTGGCAGTTACTATATATTAGTAGGATATTCGTGCCACCTGGG } \\
\text { TCCTITC }\end{array}$ \\
\hline CEN6ARS4-rv + G-ctrl & HPLC & $\begin{array}{l}\text { AAGGGCCTCGTGATACGCCTATTTTATAGGTTAATGTCATGATAATAATGGTTTCTTAGGACGGATCGCTT } \\
\text { GCCTGTAAC }\end{array}$ \\
\hline FBA1-fw + G-ctrl & HPLC & $\begin{array}{l}\text { CTAAGAAACCATTATTATCATGACATTAACCTATAAAAATAGGCGTATCACGAGGCCCTTAGTGCATGACAAAA } \\
\text { GATGAGCTAGG }\end{array}$ \\
\hline
\end{tabular}




\section{Table 3 Primers used in this study (Continued)}

\begin{tabular}{|c|c|c|}
\hline FBA1-rv + H-ctrl & HPLC & $\begin{array}{l}\text { TTACGGATATTTAACTTACTTAGAATAATGCCATTTTITGAGTTATAATAATCCTACGTAAAATCTCAAAAATGTG } \\
\text { TGGGTCATTACG }\end{array}$ \\
\hline $\mathrm{HXK2-fw}+\mathrm{B}-\mathrm{ctrl}$ & HPLC & $\begin{array}{l}\text { GAGTGTTAGAACATAATCAGTTIATCCATGGTCTATCTCTTCTTGTCGCTITITCTCCTGACGGCACCGGGA } \\
\text { AATAAACC }\end{array}$ \\
\hline $\mathrm{HXK2}-\mathrm{rV}+\mathrm{C}-\mathrm{ctrl}$ & HPLC & $\begin{array}{l}\text { AACAATAAATACGAAATCCTTATATAAGCATCTTTTACTACCAAAAAAATTTAAAATTAAGCAAGAGAAAAAA } \\
\text { ACGAGCAATTGTTAAAAG }\end{array}$ \\
\hline K.I.URA3 -fw + A-ctrl & HPLC & $\begin{array}{l}\text { GTCGACAACCCTTAATATAACTTCGTATAATGTATGCTATACGAAGTTATTAGGTCTAGAGATCCCAATACAA } \\
\text { CAGATCACGTGATC }\end{array}$ \\
\hline K.I.URA3-rV + B-ctrl & HPLC & $\begin{array}{l}\text { AGGAGAAAAAGCGACAAGAAGAGATAGACCATGGATAAACTGATTATGTTCTAAACACTCCTCAGAAGCTC } \\
\text { ATCGAACTGTCATC }\end{array}$ \\
\hline PFK1-fw + J-ctrl & HPLC & $\begin{array}{l}\text { ATATTTATAAAGGTACCCTITTAATACAATATTTATACATGTGCGTTAAATACTTTGTTCTGTCGTCTTCGT } \\
\text { GAACCATTGTC }\end{array}$ \\
\hline PFK1-rv + D-ctrl & HPLC & $\begin{array}{l}\text { TAATATIITTCTITGAAAGTACTACCCACATCCGAACATTGCCACTTACATAGCGATGTCGAGATTCCTCA } \\
\text { ATCCATACACCATTATAG }\end{array}$ \\
\hline PFK2-fw + J-ctrl & HPLC & $\begin{array}{l}\text { GAACAAAGTATTTAACGCACATGTATAAATATTGTATTAAAAGGGTACCTTAATAAATATGAGATCCGAGG } \\
\text { GACGTITATTGG }\end{array}$ \\
\hline PFK2-rv + F-ctrl & HPLC & $\begin{array}{l}\text { GAATATCCTACTAATATATAGTAACTGCCACACGAAAAACTGTGGGTGTAAAGACTATTCATAGCCATT } \\
\text { CTCTGCTGCTTTGTTG }\end{array}$ \\
\hline PGl-fw + C-ctrl & HPLC & $\begin{array}{l}\text { TIAATTTAAATITITTGGTAGTAAAAGATGCTTATATAAGGATTTCGTATTTATTGTTITCGCGACACAAT } \\
\text { AAAGTCTTCACG }\end{array}$ \\
\hline PGI-rv + D-ctrl & HPLC & $\begin{array}{l}\text { CATCGCTATGTAAGTGGCAATGTTCGGATGTGGGTAGTACTTTCAAAAGAAAAAATATTACTGAAGAAG } \\
\text { GCATACTACGCCAAG }\end{array}$ \\
\hline TPI-fw + I-ctrl & HPLC & $\begin{array}{l}\text { TTGAGAAGATGTTCTTATTCAAATTTCAACTGTTATATAGAAGAGCAAAAAATTGCCAAGCGAAAATG } \\
\text { ACGCTTGCAGTG }\end{array}$ \\
\hline \multirow[t]{2}{*}{ TPI-rv + H-ctrl } & HPLC & $\begin{array}{l}\text { ACGTAGGATTATTATAACTCAAAAAAATGGCATTATTCTAAGTAAGTTAAATATCCGTAATAGTGTGAG } \\
\text { CGGGATTTAAACTGTG }\end{array}$ \\
\hline & & For multipex $P C R$ \\
\hline A Ctrl Fw & Desalted & AAATAAACAAATAGGGGTTCCGC \\
\hline A Ctrl Rv & Desalted & GCAACACTCACTTCAACTTCATC \\
\hline B Ctrl Fw & Desalted & TTACCACCATCCAATGCAGAC \\
\hline B Ctrl Rv & Desalted & ACGGAATAGAACACGATATTTGC \\
\hline C Ctrl Fw & Desalted & TCACGGGATTTATTCGTGACG \\
\hline C Ctrl Rv & Desalted & GCGTCCAAGTAACTACATTATGTG \\
\hline D Ctrl Fw & Desalted & ACTCGCCTCTAACCCCACG \\
\hline D Ctrl Rv & Desalted & ACGGACTATAATGGTGTATGGATTG \\
\hline J Ctrl Fw & Desalted & GCTTAATCTGCGTTGACAATGG \\
\hline J Ctrl Rv & Desalted & CAATAAACGTCCCTCGGATCTC \\
\hline F Ctrl Fw & Desalted & GACGCCATTTGGACGAAAAAAAG \\
\hline F Ctrl Rv & Desalted & ATAGCACGTGATGAAAAGGAC \\
\hline G Ctrl Fw & Desalted & GCGTGTAAGTTACAGGCAAGC \\
\hline G Ctrl Rv & Desalted & GCTCTTTTCTTCTGAAGGTCAATG \\
\hline $\mathrm{HCtrl} \mathrm{FW}$ & Desalted & GTTACGTGCTCAGTTGTTAGATATG \\
\hline $\mathrm{H} C$ trl Rv & Desalted & GCAGAAGTGTCTGAATGTATTAAGG \\
\hline I Ctrl Fw & Desalted & TGAGCCACTTAAATTTCGTGAATG \\
\hline I Ctrl Rv & Desalted & GCCTITGAGTGAGCTGATACC \\
\hline
\end{tabular}




\section{Analysis by multiplex PCR}

Colonies were randomly picked and incubated overnight in appropiate medium maintaining selection pressure for the plasmid. The assemblies were isolated from $1 \mathrm{ml}$ of exponentially growing culture using the GenElute ${ }^{\mathrm{TM}}$ Plasmid Miniprep Kit by adding an extra step to the supplied protocol; after harvesting the cells by centrifugation, the pellet was resuspended in $200 \mu \mathrm{l}$ resuspension buffer supplemented with $3 \mu \mathrm{l} 1000 \mathrm{U} \cdot \mathrm{ml}^{-1}$ Zymolyase (Amsbio, Abingdon, United Kingdom) and incubated for $30 \mathrm{~min}$ at $37^{\circ} \mathrm{C}$ to digest the cell walls of the yeast cells. Further steps were performed as described by the manufacturer's recommendations. Multiplex PCR was performed with DreamTaq PCR Master Mix (2x) (Thermo Fisher Scientific). Primers were used at a concentration of $150 \mathrm{nM}$ and given in Table 3. Cycling parameters were $94^{\circ} \mathrm{C}$ for $3 \mathrm{~min}$, then 35 cycles of $94^{\circ} \mathrm{C}$ for $30 \mathrm{~s}, 55^{\circ} \mathrm{C}$ for $90 \mathrm{~s}$, and $72^{\circ} \mathrm{C}$ for $60 \mathrm{~s}$, followed by a $10 \mathrm{~min}$ incubation at $72^{\circ} \mathrm{C}$. Of each reaction $10 \mu \mathrm{l}$ was loaded on a $2 \%$ agarose gel and gel electrophoresis was performed in $0.5 \mathrm{x}$ TBE buffer at $120 \mathrm{~V}$ for $40 \mathrm{~min}$.

\section{DNA Isolation and sequencing}

One positive assembly, as determined by multiplex PCR, was transformed to Electro Ten-Blue Electro-Competent cells (Agilent Technologies, Santa Clara, CA) according to the manufacturer's instructions. From a resulting clone, plasmid DNA was isolated and analyzed by multiplex PCR. This isolated plasmid was named pUDC074. To isolate enough plasmid DNA for sequencing the plasmid was amplified in a $100 \mathrm{ml} \mathrm{E}$. coli cell culture and extracted using the method of Birnboim et al. [43] The resulting plasmid was further purified using the Zyppy Miniprep kit (Zymoresearch, Irvine, CA). For the sequencing of pUDC074, a library of 250-bp inserts was constructed and paired-end sequenced (100 base pair reads) using an Illumina HISeq 2000 sequencer (Illumina, Eindhoven, The Netherlands) provided by Baseclear BV (Leiden, The Netherlands), generating 2 million reads. A subset of 4000 randomly picked reads, which represents a 20 -fold coverage of the plasmid sequence was de novo assembled using IDBA [44]. IDBA was used with the following parameters: i) the paired end information was not used to scaffold the contig and ii) iterations were performed with k-mers ranged from $\mathrm{kmin}=21, \mathrm{kmax}=99$. One contig with a size of 20.8 kbase pair was assembled in which the ends were duplicated and could be merged into a circular sequence. Finally, the assembled contig was aligned to the in silico designed pUDC074 plasmid sequence using Clustal X in Clone Manager 9 (Sci-Ed Software, Cary, NC).

\section{Complementation studies}

The required heterozygous deletion mutants (EUROSCARF, Frankfurt, Germany) were transformed with pUDC074 and clones were screened by multiplex PCR for presence of the plasmid. For each heterozygous deletion mutant a clone containing the plasmid was transferred to solid sporulation medium and incubated for 5 days. Tetrads dissection was performed as described previously [45] using a MSM 400 micromanipulator (Singer instrument, Watchet, United Kingdom). Digested asci were plated on URA drop-out medium to rescue auxotrophy and to select for spores containing the plasmid. After incubation of 3 days, colonies were replica plated on synthetic URA dropout medium + G418 to select for presence of the KanMX marker. Plates were incubated for 2 days and checked for growth. For complementation of $H X K 2$, S. cerevisiae strain IMX188 was transformed with pUDC074. IMX188 is deficient in glucose phoshorylation. Transformants were plated on SM with glucose as the sole carbon source and incubated for 3 days. A colony was picked and checked for presence of the plasmid by multiplex PCR. The resulting strain was named IMX214. IMX188 and IMX214 were grown overnight in SM 2\% galactose. Dilutions were made for a spot plate experiment (1000, 100, $10,1$ cells $/ 10 \mu \mathrm{l})$ and $10 \mu \mathrm{l}$ of each dilution were spotted on a SM plate with either $2 \%$ glucose $(\mathrm{w} / \mathrm{v})$ or $2 \%$ galactose $(\mathrm{w} / \mathrm{v})$ as carbon source. The plates were incubated at $30^{\circ} \mathrm{C}$ for 2 days and checked for growth.

\section{Competing interests}

The authors declare that they have no competing interests.

\section{Author's contributions}

NGAK, DSE, JTP, JMD and PD-L designed the experimental work. NGAK and LB carried out the molecular biology experiments. MvdB performed the de novo sequence assembly of the plasmid. NGAK, JTP, JMD and PD-L prepared the manuscript. All authors read and approved the final manuscript.

\section{Acknowledgements}

We thank Pilar de la Torre for her technical assistance. We thank Dr. Dan Gibson (JCVI) for his kind advice during the initial phase of implementing in vivo assembly in our lab and Dr. Daniel González Ramos for critically reading this manuscript.

\section{Funding}

This work was supported by the Technology Foundation STW via a Vidi Grant (grant number 10776) to PDL; and the Kluyver Centre for Genomics of Industrial Fermentation. J.T.P. and J.-M.D. were also supported by the 'Platform Green Synthetic Biology' program (http://www.pgsb.nl/) and by BIOFLAVOUR, COST Action FA0907 (www.bioflavour.insa-toulouse.fr).

\section{Author details}

'Department of Biotechnology, Delft University of Technology, Julianalaan 67, Delft 2628 BC, The Netherlands. ${ }^{2}$ Kluyver Centre for Genomics of Industrial Fermentation, PO Box 5057, Delft 2600 GA, The Netherlands. ${ }^{3}$ Platform Green Synthetic Biology, Julianalaan 67, Delft 2628 BC, The Netherlands.

Received: 10 January 2013 Accepted: 29 April 2013 Published: 10 May 2013

\section{References}

1. Cohen SN, Chang AC, Boyer HW, Helling RB: Construction of biologically functional bacterial plasmids in vitro. Proc Natl Acad Sci U S A 1973, 70:3240-3244 
2. Mullis KB, Faloona FA: Specific synthesis of DNA in vitro via a polymerasecatalyzed chain reaction. Method Enzymol 1987, 155:335-350.

3. Li MZ, Elledge SJ: Harnessing homologous recombination in vitro to generate recombinant DNA via SLIC. Nat Methods 2007, 4:251-256.

4. Benoit RM, Wilhelm RN, Scherer-Becker D, Ostermeier C: An improved method for fast, robust, and seamless integration of DNA fragments into multiple plasmids. Protein Expres Purif 2006, 45:66-71.

5. Gibson DG, Young L, Chuang RY, Venter JC, lii CAH, Smith HO, et al: Enzymatic assembly of DNA molecules up to several hundred kilobases. Nat Methods 2009, 6:12-16.

6. Raymond CK, Pownder TA, Sexson SL: General method for plasmid construction using homologous recombination. Biotechniques 1999, 26:134-138.

7. Gibson DG, Benders G, Axelrod KC, Zaveri J, Algire M, Moodie M, et al: Onestep assembly in yeast of 25 overlapping DNA fragments to form a complete synthetic Mycoplasma genitalium genome. Proc Natl Acad Sci U S A 2008, 105:20404-20409.

8. Shao $\mathrm{Z}$, Zhao $\mathrm{H}$, Zhao $\mathrm{H}$ : DNA assembler, an in vivo genetic method for rapid construction of biochemical pathways. Nucleic Acids Res 2009, 37:e16.

9. Shao Z, Luo Y, Zhao H: Rapid characterization and engineering of natural product biosynthetic pathways via DNA assembler. Mol BioSystems 2011, 7:1056-1059.

10. Resnick M, Martin P: The repair of double-strand breaks in the nuclear DNA of Saccharomyces cerevisiae and its genetic control. Mol Gen Genet 1976, 143:119-129.

11. Ma H, Kunes S, Schatz PJ, Botstein D: Plasmid construction by homologous recombination in yeast. Gene 1987, 58:201-216.

12. Larionov V, Kouprina N, Graves J, Resnick MA: Highly selective isolation of human DNAs from rodent-human hybrid cells as circular yeast artificial chromosomes by transformation-associated recombination cloning. Proc Natl Acad Sci U S A 1996, 93:13925-13930.

13. Raymond CK, Olson MV, Sims EH: Linker-mediated recombinational subcloning of large DNA fragments using yeast. Genome Res 2002, 12:190-197.

14. Gibson DG, Benders G, Andrews-Pfannkoch C, Denisova E, Baden-Tillson H, Zaveri J, et al: Complete chemical synthesis, assembly, and cloning of a Mycoplasma genitalium genome. Science 2008, 319:1215-1220.

15. Gibson DG: Synthesis of DNA fragments in yeast by one-step assembly of overlapping oligonucleotides. Nucleic Acids Res 2009, 37:6984-6990.

16. Gibson DG: Gene and genome construction in yeast. Curr Protoc Mol Biol 2011, 94:3.22.1-3.22.17.

17. Shao Z, Luo Y, Zhao H: DNA assembler method for construction of zeaxanthin-producing strains of Saccharomyces cerevisiae. Methods $\mathrm{Mol}$ Biol 2012, 898:251-262

18. Muller H, Annaluru N, Schwerzmann JW, Richardson SM, Dymond JS, Cooper EM, et al: Assembling large DNA segments in yeast. Methods Mol Biol 2012, 852:133-150

19. Raymond CK, Sims EH, Olson MV: Linker-mediated recombinational subcloning of large DNA fragments using yeast. Genome Res 2002, 12:190-197.

20. Anderson PR, Haj-Ahmad Y: Counter-selection facilitated plasmid construction by homologous recombination in Saccharomyces cerevisiae. Biotechniques 2003, 35:692-694. 696, 698.

21. Fishman-Lobell J, Rudin N, Haber JE: Two alternative pathways of doublestrand break repair that are kinetically separable and independently modulated. Mol Cell Biol 1992, 12:1292-1303.

22. Boulton S: Identification of a Saccharomyces cerevisiae Ku80 homologue: roles in DNA double strand break rejoining and in telomeric maintenance. Nucleic Acids Res 1996, 24:4639-4648.

23. Manivasakam P, Weber SC, McElver J, Schiestl RH: Micro-homology mediated PCR targeting in Saccharomyces cerevisiae. Nucleic Acids Res 1995, 23:2799-2800.

24. Suzuki K, Imai Y, Yamashita I, Fukui S: In vivo ligation of linear DNA molecules to circular forms in the yeast Saccharomyces cerevisiae. J Bacteriol 1983, 155:747-754

25. Shoemaker DD, Lashkari DA, Morris D, Mittmann M, Davis RW: Quantitative phenotypic analysis of yeast deletion mutants using a highly parallel molecular bar-coding strategy. Nat Genet 1996, 14:450-456.

26. Nijkamp JF, Van Den Broek M, Datema E, de Kok S, Bosman L, Luttik M, et al: De novo sequencing, assembly and analysis of the genome of the laboratory strain Saccharomyces cerevisiae CEN.PK113-7D, a model for modern industrial biotechnology. Microb Cell Fact 2012, 11:36.

27. Altschul SF, Gish W, Miller W, Myers EW, Lipman DJ: Basic local alignment search tool. J Mol Biol 1990, 215:403-410.

28. Aguilera A: Deletion of the phosphoglucose isomerase structural gene makes growth and sporulation glucose dependent in Saccharomyces cerevisiae. Mol Gen Genet 1986, 204:310-316.

29. Compagno C, Brambilla L, Capitanio D, Boschi F, Ranzi BM, Porro D: Alterations of the glucose metabolism in a triose phosphate isomerasenegative Saccharomyces cerevisiae mutant. Yeast 2001, 18:663-670.

30. Schwelberger HG, Kohlwein SD, Paltauf F: Molecular cloning, primary structure and disruption of the structural gene of aldolase from Saccharomyces cerevisiae. Eur J Biochem 1989, 180:301-308.

31. Breitenbach-Schmitt I, Heinisch J, Schmitt HD, Zimmermann FK: Yeast mutants without phosphofructokinase activity can still perform glycolysis and alcoholic fermentation. Mol Gen Genet 1984, 195:530-535.

32. Oldenburg KR, Vo KT, Michaelis $S$, Paddon C: Recombination-mediated PCR-directed plasmid construction in vivo in yeast. Nucleic Acids Res 1997, 25:451-452.

33. van Dijken JP, Bauer J, Brambilla L, Duboc P, Francois JM, Gancedo C, et al: An interlaboratory comparison of physiological and genetic properties of four Saccharomyces cerevisiae strains. Enzyme Microb Technol 2000, 26:706-714

34. Shao Z, Luo Y, Zhao H: DNA assembler method for construction of zeaxanthin-producing strains of Saccharomyces cerevisiae. Method Mol Biol 2012, 898:251-262

35. Noskov VN, Koriabine M, Solomon G, Randolph M, Barrett JC, Leem SH, et al: Defining the minimal length of sequence homology required for selective gene isolation by TAR cloning. Nucleic Acids Res 2001, 29:E32.

36. Ro DK, Paradise EM, Ouellet M, Fisher KJ, Newman KL, Ndungu JM, et al: Production of the antimalarial drug precursor artemisinic acid in engineered yeast. Nature 2006, 440:940-943.

37. Shetty RP, Endy D, Knight TF Jr: Engineering BioBrick vectors from BioBrick parts. J Biol Eng 2008, 2:5.

38. Verduyn C, Postma E, Scheffers W, van Dijken JP: Effect of benzoic acid on metabolic fluxes in yeasts: a continuous-culture study on the regulation of respiration and alcoholic fermentation. Yeast 1992, 8:501-517.

39. Pronk JT: Auxotrophic yeast strains in fundamental and applied research. Appl Environ Microbiol 2002, 68:2095-2100.

40. Gueldener U, Heinisch J, Koehler GJ, Voss D, Hegemann JH: A second set of loxP marker cassettes for Cre-mediated multiple gene knockouts in budding yeast. Nucleic Acids Res 2002, 30:e23.

41. Sikorski RS, Hieter P: A system of shuttle vectors and yeast host strains designed for efficient manipulation of DNA in Saccharomyces cerevisiae. Genetics 1989, 122:19-27.

42. Gietz RD, Woods R: Transformation of yeast by lithium acetate/singlestranded carrier DNA/polyethylene glycol method. Method Enzymol 2002, 350:87-96.

43. Birnboim HC, Doly J: A rapid alkaline extraction procedure for screening recombinant plasmid DNA. Nucleic Acids Res 1979, 7:1513-1523.

44. Peng $Y$, Leung $H$, Yiu SM, Chin F: IDBA - practical iterative de Bruijn graph de novo assembler. In Research in Computational Molecular Biology. 6044th edition. Edited by Berger B. Berlin: Springer; 2010:426-440.

45. Burke D, Dawson D, Stearns T: Methods in Yeast Genetics: a Cold Spring Harbor Laboratory course manual. 2000th edition. Cold Spring Harbor, NY: Cold Spring Harbor Laboratory Press; 2000

doi:10.1186/1475-2859-12-47

Cite this article as: Kuijpers et al:: A versatile, efficient strategy for assembly of multi-fragment expression vectors in Saccharomyces cerevisiae using 60 bp synthetic recombination sequences. Microbial Cell Factories 2013 12:47. 